\title{
Alterstice
}

Revue internationale de la recherche interculturelle

International Journal of Intercultural Research

Revista International de la Investigacion Intercultural

\section{Le débat sur le voile à l'école à la lumière des diverses conceptions de l'ethnicité et des rapports ethniques}

\section{Marie Mc Andrew}

\section{Volume 1, numéro 1, 2011}

Penser la recherche interculturelle : le défi des diversités

URI : https://id.erudit.org/iderudit/1077588ar

DOI : https://doi.org/10.7202/1077588ar

Aller au sommaire du numéro

Éditeur(s)

Alterstice

ISSN

1923-919X (numérique)

Découvrir la revue

Citer cet article

Mc Andrew, M. (2011). Le débat sur le voile à l'école à la lumière des diverses conceptions de l'ethnicité et des rapports ethniques. Alterstice, 1(1), 19-33. https://doi.org/10.7202/1077588ar

\section{Résumé de l'article}

Dans plusieurs sociétés multiethniques, la légitimité de permettre aux jeunes musulmanes de porter le traditionnel voile islamique ou hijab au sein des établissements scolaires publics a suscité des débats passionnés. Le propos de cet article est de se pencher de nouveau sur différents cas nationaux de controverses autour du port du hijab, à partir de la perspective de la sociologie des rapports ethniques. L'auteure explore d'abord les limites des conceptions univoques de l'ethnicité, qu'elles soient essentialistes, hétérocentristes ou individualistes, tant du point de vue théorique que pratique, lorsqu'on les applique à un enjeu de politique comme l'accommodement de la diversite religieuse dans les écoles publiques et ses relations à l'égalité des sexes. Dans un second temps, elle fait valoir que seule une perspective multivoque sur la construction des frontières ethniques et une analyse contextuelle peuvent refléter la complexité des dynamiques que soulèvent de telles controverses. Finalement, en conclusion, elle propose des balises sur les principes qui devraient guider l'action publique à cet égard. 


\section{ज alterstice \\ ARTICLE THÉMATIQUE \\ Le débat sur le voile à l'école à la lumière des diverses conceptions de l'ethnicité et des rapports ethniques}

Marie Mc Andrew ${ }^{1}$

\section{Résumé}

Dans plusieurs sociétés multiethniques, la légitimité de permettre aux jeunes musulmanes de porter le traditionnel voile islamique ou hijab au sein des établissements scolaires publics a suscité des débats passionnés. Le propos de cet article est de se pencher de nouveau sur différents cas nationaux de controverses autour du port du hijab, à partir de la perspective de la sociologie des rapports ethniques. L'auteure explore d'abord les limites des conceptions univoques de l'ethnicité, qu'elles soient essentialistes, hétérocentristes ou individualistes, tant du point de vue théorique que pratique, lorsqu'on les applique à un enjeu de politique comme l'accommodement de la diversité religieuse dans les écoles publiques et ses relations à l'égalité des sexes. Dans un second temps, elle fait valoir que seule une perspective multivoque sur la construction des frontières ethniques et une analyse contextuelle peuvent refléter la complexité des dynamiques que soulèvent de telles controverses. Finalement, en conclusion, elle propose des balises sur les principes qui devraient guider l'action publique à cet égard.

\section{Rattachement de l'auteure \\ ${ }^{1}$ Département d'administration et fondements de l'éducation, Faculté des sciences de l'éducation, Chaire de recherche du Canada sur l’Éducation et les rapports ethniques, Université de Montréal, Montréal, Canada}

\section{Correspondance}

marie.mcandrew@umontreal.ca

\section{Mots clés}

ethnicité; voile; actions publiques; relations ethniques

\section{Pour citer cet article :}

Mc Andrew, M. (2011). Le débat sur le voile à l'école à la lumière des diverses conceptions de l'ethnicité et des rapports ethniques. Alterstice, 1(1), 19-34. 


\section{Introduction}

Dans plusieurs sociétés multiethniques, la légitimité de permettre aux jeunes musulmanes de porter le traditionnel voile islamique ou hijab au sein des établissements scolaires a suscité des débats passionnés. Le cas le plus connu est celui de la France où, depuis 1989, les « affaires du foulard » sont presque devenues un sport national. Dans ce contexte, la controverse (Amiraux, 2010a; Gautherin, 2000; Lorcerie, 1996; Stasi, 2003) oppose les partisans d'une conception traditionnelle de la laïcité, définie comme une stricte neutralité de l'espace public, et les partisans d'une laïcité renouvelée, qui accepte l'expression de la diversité religieuse par des individus fréquentant des institutions laïques. C'est actuellement la première conception qui prévaut, avec l'adoption de la Loi $n^{\circ} 2004-228$ du 15 mars 2004 encadrant le port de signes ou de tenues manifestant une appartenance religieuse dans les établissements scolaires publics (Ministère de l'Éducation Nationale, de l'Enseignement Supérieur et de la Recherche, 2004). Mais d'autres sociétés, comme la Belgique, l'Allemagne, l'Espagne, le Canada et tout particulièrement le Québec (Mc Andrew, 2001, 2010; Milot et Koussens, 2009; Shadid et Van Koningsvel, 1996) ont également vu le personnel scolaire, les politiciens, les médias et l'opinion publique s'affronter dans diverses controverses, parfois simplistes et parfois étonnamment complexes. Celles-ci concernent, entre autres, la relation entre l'égalité des sexes et la diversité religieuse, ainsi que les balises légitimes qu'un État démocratique et son système scolaire peuvent imposer à l'expression de la diversité, autant par les majorités que par les minorités.

Pour comprendre l'intensité des émotions que soulève cette question, et surtout dégager des pistes d'action pour les décideurs et les intervenants, le recours au paradigme interculturel traditionnel s'avère limité, et ce, tant sur le plan descriptif que sur le plan normatif. D'une part, en effet, même si elles sont influencées par des contextes nationaux spécifiques, les croyances religieuses sont difficilement réductibles à de simples enjeux culturels. Le recours à la transcendance comme logique de légitimation leur donne en effet un caractère absolu qui les rend peu susceptibles de faire l'objet d'une évolution interne ou d'un compromis social, éléments qui sont au cœur du projet interculturel (Boyd, 2007; Dortier et Testo, 2005; Milot, 2009). D'autre part, l'interculturalisme comme mode de gestion des rapports collectifs rencontre des obstacles spécifiques lorsque les conflits se font à propos d'enjeux religieux (Bramadat et Seljak, 2009; Germain, Liégeois et Hoernig, 2007; Milot, Portier et Willaime, 2010). La marge de manœuvre des décideurs est plus étroite, puisque le droit à la liberté religieuse et à l'expression des croyances sont des droits fondamentaux, contrairement au droit des minorités à maintenir leur vie culturelle, plus clairement limité en fonction des législations nationales et des ressources disponibles (Bosset, 2010). De plus, les débats autour de la prise en compte de la diversité religieuse portent la plupart du temps sur des questions politiques que le simple " dialogue entre cultures » est peu susceptible de résoudre, comme l'islamophobie, le fondamentalisme masqué sous la ferveur religieuse, la nature sexiste de toutes les religions, ou encore les dangers du communautarisme (Amiraux, 2010b; Bouchard et Taylor, 2008; Milot et Koussens, 2009).

Dans cet article, j'utiliserai donc peu le paradigme interculturel, si ce n'est pour signaler, au fur et à mesure des diverses conceptions débattues, l'importance que les arguments de nature culturelle prennent. En effet, les analyses que je mène depuis plus de quinze ans (Mc Andrew, 1996, 2001, 2010; Mc Andrew, Milot, Imbeault et Eid, 2008) m'ont amenée à prendre conscience du fait que les positions adoptées de part et d'autre lors de tels débats présentent souvent la même limite: assumer une conception particulière de l'ethnicité, mais sans la rendre explicite.

Le propos de cet article est plutôt de revisiter la question du voile à l'école à partir de la perspective de la sociologie des rapports ethniques. Pour ce faire, je me fonde sur une typologie développée antérieurement (Mc Andrew, 2003a) où les diverses conceptions de la nature de l'ethnicité sont regroupées en quatre grands idéaux types à partir de la littérature scientifique et de l'analyse de contenu des prises de position de divers acteurs sociaux. La démarche ne vise pas à renouveler la réflexion théorique sur la nature de l'ethnicité, ni à décrire ce champ de manière exhaustive. Il s'agit plutôt d'illustrer, de manière heuristique, comment le fait de reconnaître l'existence de conceptions multiples à cet égard, et ce même chez des personnes qui se réclament d'un paradigme constructiviste commun, permet d'éclairer les positions adoptées dans le débat public. Dans un premier temps, j'explorerai les limites des conceptions univoques de l'ethnicité, qu'elles soient essentialistes, hétérocentristes ou individualistes, tant du point de vue théorique que pratique, pour répondre à l'enjeu de l'accommodement de la diversité 
religieuse dans les écoles publiques et ses relations avec l'égalité des sexes. Dans un second temps, je ferai valoir que seule une perspective multivoque sur la construction des frontières ethniques et une analyse contextuelle peuvent refléter la complexité des dynamiques que soulèvent de telles controverses. Finalement, en me fondant sur une telle perspective, je proposerai un ensemble de balises qui devraient guider l'action publique à cet égard.

\section{Les limites des conceptions univoques de l'ethnicité}

\section{L'essentialisme culturel : le hijab comme un non-enjeu}

Le maintien paradoxal de l'ethnicité durant tout le $X X^{e}$ siècle, voire l'intensification de son importance, a été interprété à partir de différentes perspectives théoriques. Au sein de la communauté universitaire, seule une minorité d'analystes (Gil-White, 2001; Van Den Berghe, 1967) y auront vu la justification d'un paradigme essentialiste où le maintien des groupes ethniques est expliqué principalement par leurs différences culturelles. Aujourd'hui, l'essentialisme est presque agonisant, sous l'effet cumulé des critiques multidisciplinaires auxquelles il a été soumis depuis une trentaine d'années.

Les psychologues sociaux nous ont d'abord rappelé, à partir de nombreuses études expérimentales, que l'identité ethnique est un phénomène mouvant et que sa "saillance " chez un individu par rapport à d'autres identités attribuées (genre, classe sociale) ou volontaires (style de vie, groupe professionnel) est essentiellement situationnelle (Bourhis et Harvey, 2010; Bourhis et Leyens, 1994; Taboada-Leonetti, 1990; Tajfel, 1982). Ils ont également illustré que la différence culturelle n'est pas ce qui constitue l'identité ethnique, mais plutôt l'inverse : les différences intragroupes sont systématiquement sous-évaluées et les différences intergroupes surévaluées dans le processus de construction des frontières par les individus pour lesquels l'appartenance ethnique ou nationale est primordiale.

Un autre courant critique (Barth, 1969; Bilge, 2010; Guillaumin, 1977; Juteau, 2000), basé sur la sociologie et l'anthropologie, attaque davantage le caractère fixiste et a-historique des perspectives essentialistes sur l'ethnicité. Il fait, entre autres, valoir que même le commun des mortels - et cela va sans dire des analystes universitaires peuvent constater presque quotidiennement comment la redéfinition des marqueurs culturels de divers groupes sont loin de toujours s'accompagner de redéfinition des frontières qui les séparent (Juteau, 1993).

Finalement, l'essentialisme culturel a aussi été critiqué à partir d'une perspective normative issue de la philosophie politique, qui a fait valoir les dangers de dérives antidémocratiques d'une attribution non critique des individus à leur groupe d'origine (Kymlicka, 1995, 2000; Touraine, 1997). La critique philosophique s'inscrit aussi dans la foulée de la réflexion juridique sur la reconnaissance du pluralisme, qui considère ce dernier essentiellement comme un droit porté par des individus et non des groupes, du moins en ce qui concerne les minorités immigrantes (Weinstock, 2007; Woehrling, 2008).

Comme on le constate, il n'est pas recommandé aujourd'hui, du moins dans les cercles universitaires et gouvernementaux, d'être taxé d'essentialiste culturel. La perspective dominante est, en effet, celle du constructivisme, qui aborde l'identité ethnique comme un phénomène dynamique, que ce soit d'un point de vue analytique, dans ses dimensions individuelles ou collectives, ou d'un point de vue normatif. Cependant, on peut constater chaque jour que pour les gens ordinaires, et même au sein du discours politique, l'essentialisme culturel est un système très important, sinon dominant, d'interprétation de l'ethnicité (Beauchemin, 2003; Buyck et Fall, 1995; Helly, 1992; Potvin, 2008). En effet, la plupart des individus vivent leur appartenance à un groupe ou à une culture spécifique comme un phénomène relativement stable. S'ils peuvent être ouverts à remettre en question cet a priori lorsqu'on les expose à certaines des critiques exprimées plus haut, dans la vie quotidienne, leur appartenance ethnique est souvent assumée comme un "en-soi ». Cette résistance est d'ailleurs largement documentée lors des ateliers et des formations à visée interculturelle et lors de leur évaluation subséquente (Hohl et Cohen-Émerique, 1999; Kanouté, Lavoie et Duong, 2004; Verlot, 2002).

De plus, lorsqu'on aborde les débats relatifs à la scolarisation, l'essentialisme culturel est encore plus populaire et ce, tout particulièrement chez les minorités. Le contrôle d'institutions spécifiques est un des thèmes dominants au sein de ce courant, qui s'attache essentiellement à la fonction de reproduction culturelle de l'école (Glenn et De 
Jong, 1996; Gallagher, 2004; Holmes, 1981). L'ethnicité est considérée comme un contenu à reproduire le plus fidèlement possible face à un groupe majoritaire auquel on attribue également une culture cohérente ainsi qu'une intention univoque d'assimilation. La transformation des frontières ethniques ou la création d'une nouvelle culture hybride n'est clairement pas à l'ordre du jour dans certains milieux.

Dans une telle perspective, la question du hijab est considérée comme un non-enjeu (Brodeur, 2010; Leblanc, Fortin et Le Gall, 2008; Milot, 2009; Venel, 2004). Les personnes qui ont une conception essentialiste de l'ethnicité n'arrivent pas à comprendre l'existence d'un tel débat. Le hijab est considéré comme partie intégrante de la religion et de la culture, et le rôle des écoles publiques à cet égard, comme évident : respecter le désir des parents. Cette position est très bien illustrée par la réaction de certains musulmans de Toronto à la controverse québécoise de 1995, qui a amené le Globe and Mail à publier un article intitulé « My hijab is... none of your business » (Kurd, 1995). Pour les musulmans les plus pratiquants, toutefois, la tolérance au port du hijab à l'école publique est souvent un "second choix " : serait préférée la création - et surtout le financement - d'institutions spécifiques dont l'ethos refléterait de manière beaucoup plus exhaustive l'ensemble des valeurs religieuses (Forum musulman canadien, 1999; Halstead, 1986; Sarwar, 1994).

Toutefois, l'essentialisme culturel est aussi très répandu au sein des groupes majoritaires. On en rencontre parfois une version multiculturelle édulcorée qui amène ses partisans à appuyer la tolérance du port du hijab à partir des mêmes arguments que les membres des minorités religieuses. Mais les partisans du racisme différentiel peuvent aussi trouver dans cette perspective une confirmation de leur croyance dans l'incompatibilité de diverses cultures (Potvin, 2008; Taguieff, 1987) et, dans certains cas, ils se réjouiront des demandes des minorités en faveur de davantage de ségrégation en éducation.

Bien que cette vision ait un fort pouvoir de séduction sur les individus qui partagent cette croyance, les limites de l'essentialisme culturel comme système d'interprétation ou de justification du port du hijab à l'école publique sont évidentes. Tout d'abord, une telle perspective néglige totalement les contradictions potentielles pour les jeunes filles musulmanes entre leur identité de genre et leur identité ethnique. On suppose également que tous les individus appartiennent de façon non critique à leur groupe d'origine ou à leur religion familiale, et que les mineures, au lieu d'être activement engagées dans un processus de construction de leur propre identité, devraient essentiellement refléter les désirs de leurs parents. Finalement, les partisans d'une telle perspective refusent d'examiner de façon critique la pertinence de préoccupations à plus long terme concernant l'impact potentiellement négatif de la socialisation religieuse sur l'égalité des sexes, et de la promotion d'identités religieuses rigides sur la qualité des relations interculturelles.

\section{L'ethnicité comme produit de la frontière externe}

Rejetant cette conception de l'ethnicité comme un "en-soi", la perspective constructiviste s'intéresse aux frontières entre les groupes, aux marqueurs culturels qu'ils utilisent pour légitimer leur spécificité et à la fluctuation de ces deux phénomènes (Jenkins, 2008; Juteau, 2000; Rex, 2006). À cet effet, l'analyse des différences matérielles et symboliques au sein de différents champs sociaux, entre autres en éducation, ainsi que l'identification des intérêts contradictoires de différents groupes et sous-groupes, tels les élites ethniques (Vermeulen et Govers, 1996), sont essentielles. Cependant, au-delà de ce large consensus, différentes versions du constructivisme comme système d'explication des rapports ethniques ont été mises de l'avant ces quinze dernières années.

Une première conception (Fekete, 2006; Freitag, 1981; Zylberberg, 1994) peut être caractérisée comme "l'ethnicité, produit de la frontière externe ». On y insiste sur le rôle central que joue le groupe dominant par son regard, son contrôle du discours et, surtout, son pouvoir sociopolitique et économique, dans le maintien des frontières ethniques. Selon ce courant, la persistance des identités ethniques doit d'abord et avant tout être interprétée à travers le prisme des inégalités. La croyance qu'ont les individus d'appartenir à des groupes distincts ou d'afficher des différences culturelles est souvent interprétée comme une idéologie, au sens marxiste de ce terme, qui les aliènerait de leurs véritables intérêts. 
Il existe, à l'intérieur même de ce courant, deux visions différentes. La perspective marxiste (Gimenez, 2001; Hall, 1980) n'exclut pas la réalité des appartenances groupales, mais considère que la priorité doit être donnée aux solidarités de classes, l'ethnicité représentant une arme que l'ensemble des élites, qu'elles appartiennent aux groupes majoritaires ou minoritaires, utilisent pour maintenir leur pouvoir sur les classes dominées. La seconde perspective, "républicaine ", (Baubérot, 2007; Juteau, 2006; Schnapper, 1991) délégitime l'adhésion aux solidarités intermédiaires entre l'État et le citoyen, et fait de la persistance de l'ethnicité, au mieux, l'un des symptômes de l'échec d'un projet authentiquement intégrateur.

Bien que le constructivisme hétérocentriste ait l'avantage de mettre l'accent sur l'importance de la variable socioéconomique et des relations de pouvoir inégal dans la production de l'ethnicité, il présente plusieurs limites. Tout d'abord, ce courant confine au conspirationnisme, accordant à l'État un pouvoir quasi absolu dans la production des identités ethniques (Fontaine, 1993). De plus, il sous-estime fortement la frontière interne de la production de l'ethnicité, c'est-à-dire la mémoire historique et l'attachement des acteurs eux-mêmes et des appartenances spécifiques. En insistant uniquement sur l'une des facettes de la production de l'ethnicité, les "égalitaristes» marxistes ou républicains se trouvent donc souvent dans la situation paradoxale et quelque peu paternaliste de prétendre libérer malgré eux les « opprimés » qu’ils défendent.

Ces caractéristiques sont également visibles dans la position que les partisans d'un constructivisme hétérocentriste adoptent par rapport à la scolarisation. Le mandat de l'école à l'égard des élèves des groupes minoritaires est d'abord et avant tout défini en termes d'égalité des chances et de luttes contre les inégalités (Giroux, 1981; Lorcerie, 2003; Payet et Giuliani, 2010). Puisque l'ethnicité est largement appréhendée comme illusion, une idéologie au sens marxiste du terme, on ne considère pas que l'école ait à consolider sa reproduction et on sousestime fortement la réalité des luttes qui peuvent opposer groupes majoritaires et groupes minoritaires pour la définition du curriculum.

Si la fonction de socialisation de l'école est sollicitée, c'est bien davantage dans un objectif de transformation sociale et de production d'identités communes que dans un objectif de maintien et de respect du pluralisme. $Y$ domine l'éducation civique traditionnelle, où la diversité ethnoculturelle est abordée comme un obstacle à dépasser dans le processus de construction d'une société égalitaire et fraternelle (Mc Andrew, Cicéri et Jacquet, 1997).

Lorsqu'ils ont à répondre aux demandes concernant le respect des prescriptions religieuses dans les écoles publiques, les constructivistes hétérocentristes peuvent réagir de deux façons, selon l'importance respective qu'ils accordent aux inégalités intra- ou inter-groupes en matière de relations ethniques. Dans le premier cas, le port du hijab par des jeunes femmes musulmanes (qui, de façon révélatrice est souvent nommé la persistance du port du voile) est interprété essentiellement comme une posture réactive, le symbole de «l'échec de l'intégration véritable ». Cette interprétation, qui a dominé le débat français (Cicéri, 1999; Lorcerie, 2004; Stasi, 2003), et ce, autant chez les partisans de la tolérance que de l'interdiction, est en partie fondée sur des réalités sociologiques, tant sur le plan national qu'international. II serait, en effet, difficile de nier que le racisme, vécu personnellement ou collectivement par le biais de l'islamophobie ambiante, joue un rôle dans la décision de certaines jeunes filles. Mais il serait également fortement paternaliste d'affirmer que les motivations religieuses des individus qui appartiennent aux groupes minoritaires sont de simples sous-produits de leurs relations avec les groupes dominants. De plus, une telle position donne très peu d'indications concrètes aux décideurs sur la décision à prendre. Les facteurs à l'origine d'un phénomène ne nous indiquent pas s'il faut s'en réjouir ou s'en désoler.

La situation est plus facile pour ceux qui mettent l'accent sur les relations de pouvoir intra-groupes (Benhabib, 2011; Geadah, 1996; Kepel, 1992). En effet, si le port du hijab est un effet de la domination des hommes musulmans sur les femmes musulmanes ou des élites cléricales faisant la promotion d'une définition fondamentaliste de l'islam aux dépens des interprétations plus modérées, il devient extrêmement difficile de faire la promotion de la tolérance d'une telle pratique. Le thème du hijab comme « un complot sexiste ou fondamentaliste " a donc un impact extrêmement puissant auprès de l'opinion publique, et ce, que les politiques officielles favorisent ou interdisent l'expression de la diversité religieuse au sein des écoles publiques (Amara, 2003; Amiraux, 2010a; Bernard, 1995; Gaudet, 1994). 
Mais, ici encore, le danger de réductionnisme et de paternalisme est évident. Beaucoup de pratiques culturelles ou de croyances partagées par les citoyens d'origines diverses sont les produits partiels de relations inégales ou d'une acceptation non critique de l'influence de différentes institutions comme les Églises, les clubs, les partis politiques ou les médias. Cependant, à moins que des contraintes physiques ou morales ne soient en cause, on considère généralement qu'on doit respecter la liberté des individus de faire des choix que d'autres, aussi éclairés soient-ils, pourraient considérer comme non fondés (Beiner, 1995; Kymlicka, 1995). Une telle position reconnaît que la motivation à agir est toujours complexe et qu'elle ne peut être réduite aux seules inégalités qui ont pu l'influencer. Les constructivistes hétérocentristes demandent souvent qu'on applique aux revendications des groupes ethniques, et plus particulièrement à celles des musulmans, un ensemble de règles différentes de celles qu'on utilise habituellement dans les régimes démocratiques pour statuer si une pratique est acceptable ou non. À moins que l'on accepte leur argument selon lequel l'ethnicité est un pur sous-produit d'une oppression interne ou externe, il est très difficile de les suivre dans cette voie.

\section{L'ethnicité comme produit et prérogative de l'individu : le hijab comme choix individuel}

Une seconde école de pensée, relevant également d'une perspective constructiviste, peut être définie comme celle de "l'ethnicité, produit et prérogative de l'individu ». Fortement influencée par l'interactionnisme social (Berger et Luckman, 1967), ses tenants s'intéressent essentiellement aux processus et interactions à travers lesquels l'individu est amené à croire qu'il est différent, et à définir ses appartenances.

Dans une version qu'on pourrait qualifier de moderne (Bourgeault, Gagnon, Mc Andrew et Pagé, 1995), puisqu'elle accorde encore un rôle unificateur au Sujet à cet égard et correspond largement au discours du libéralisme en philosophie politique, on insiste sur l'autonomie morale de l'individu qui, face à des influences diverses et des sollicitations multiples liées à la variété de ses allégeances sociales ou individuelles, construit lui-même sa formule culturelle, certains employant d'ailleurs à cet égard l'expression révélatrice de " bricolage identitaire " (Camilleri et collab., 1990; Deschamps et Devos, 1999; Laperrière, 1991). C'est souvent ce paradigme qui domine chez les tenants de l'interculturalisme, tant au niveau analytique que normatif. Dans une version plus radicale, qu'on peut qualifier de postmoderne si l'on considère que le projet postmoderniste est précisément la dissolution du Sujet, l'ethnicité est plus ou moins réduite à une des « narratives » qui traversent la conscience éclatée et en perpétuelle redéfinition d'un Sujet de moins en moins producteur de sens (Burawoy et collab., 2000; Hoerder, 2000; Sarup, 1988).

La principale force d'une telle perspective est de redonner aux acteurs de terrain une place importante face aux déterminismes sociaux. Cependant, cette idéologie paraît souvent relever d'un volontarisme quelque peu naïf, comme si les sujets construisaient leur identité ou leur "récit ", selon le cas, en dehors des grands rapports sociaux. Pour reprendre la formule d'Orwell, les constructivistes individualistes semblent parfois ignorer le fait que si tous les individus sont libres de choisir leurs identités, certains sont plus libres que d'autres à cet égard (Probyn, 1987)! De plus, ils sous-estiment parfois l'autonomie et la pérennité des appartenances groupales incarnées dans une histoire et dans des institutions propres, comme si les identités et les appartenances étaient essentiellement des produits que l'individu peut facilement décider de mettre de côté.

Pour les tenants de cette approche, c'est d'abord la fonction de l'école dans la production/reproduction culturelle qui est centrale. En effet, l'école, lieu unique de socialisation intensive entre pairs de toutes origines à l'âge où se forment les identités et les attitudes, est considérée comme le laboratoire où se redéfinissent à chaque génération les frontières ethniques (Abdallah-Pretceille, 1992; Gallagher, 2004; Guttman, 1987). Puisque la liberté et l'autonomie morale de l'individu doivent être préservées, la résultante souhaitable de ce courant n'est pas définie a priori. Les partisans de ce courant ne valorisent ni ne combattent le pluralisme ou l'assimilation. Leur priorité est celle de l'interaction, ce qui, sur le plan des politiques et des programmes scolaires, les amènent à privilégier des institutions communes au sein desquelles la négociation interculturelle est ouverte. Les conséquences d'une telle approche sur la reproduction culturelle des groupes dominants et des groupes dominés sont rarement débattues et, si les inégalités individuelles sont susceptibles d'être reconnues lorsqu'elles émergent à travers l'interaction au sein du système scolaire, l'absence d'une analyse critique à caractère plus macrosociologique de la fonction de sélection de l'école se fait souvent sentir (Bourgeault et Pietrantonio, 1996; Dei, 1996).

Alterstice - Revue Internationale de la Recherche Interculturelle, vol. 1, $n^{\circ} 1$ 
Les constructivistes individualistes sont généralement partisans de l'accommodement des pratiques religieuses à l'intérieur des espaces publics, dans la mesure où les revendications à cet égard sont basées sur des choix personnels. Ils s'opposent toutefois à l'imposition par la communauté d'une croyance non partagée par l'individu, et ce, que ce soit dans des institutions publiques ou ethno-spécifiques. En établissant cette distinction, ils se situent clairement dans la foulée du libéralisme renouvelé, qui met de l'avant comme seule limite légitime à la reconnaissance de la diversité l'équilibre des droits et le respect des valeurs démocratiques (Bosset, 2010; Bourgeault, Gagnon, Mc Andrew et Pagé, 1995; Kymlicka et Norman, 2000). Le « hijab, choix individuel » est une conception extrêmement populaire parmi les partisans de la tolérance, qu'ils appartiennent aux groupes majoritaires ou minoritaires (Bouchard et Taylor, 2008; Commission des droits de la personne du Québec, 1995; Commission ontarienne des droits de la personne, 1996; Ramadan, 1994). Le fait que l'interaction sociale soit si importante pour les partisans d'une telle approche explique leur inclination au compromis, parce que celui-ci permet de préserver le partage d'institutions communes.

Aussi attirante que cette perspective puisse sembler, elle n'est cependant pas sans limites, spécialement dans le champ éducatif. Tout d'abord, définir ce qui constitue un choix personnel authentique et le type de contexte au sein duquel celui-ci peut être exercé est une tâche difficile (Hargreaves, 1996). Dans la foulée des limites de l'interactionnisme social, la toile complexe de contraintes liées aux relations de pouvoir internes et externes auxquelles font face les femmes musulmanes lorsqu'elles ont à décider de porter ou non le hijab est souvent insuffisamment reconnue (Bilge, 2008, 2010). Face à cette complexité, certains groupes de pression musulmans utilisent le concept du "libre-choix " avec un tel laxisme qu'il en est souvent réduit à une simple rhétorique (Gautherin, 2000; Milot, 2010). Quant aux opposants à cette pratique, ils mettent souvent le seuil si haut que la plupart des choses que nous faisons en société, largement influencées par notre héritage culturel et notre socialisation, ne seraient pas en mesure de répondre à leurs exigences (Bosset, 2010; Boyd, 2007; Cicéri, 1999).

De plus, la controverse autour du port du hijab à l'école ne concerne pas des adultes dont on peut affirmer qu'ils exercent leur indépendance morale, mais des enfants et des adolescents qui sont en train d'apprendre à exercer leurs droits et à définir leur propre système de croyances. Les conflits sur les pratiques religieuses opposent donc, la plupart du temps, deux groupes d'adultes : les parents et le personnel scolaire qui, l'un et l'autre, mettent de l'avant leur propre définition du bien de l'enfant (Mc Andrew, 2003b). Ces deux parties ont un rôle légitime à jouer qu'il n'est pas facile d'arbitrer. Les parents, majoritaires comme minoritaires, sont légalement les porteurs des droits des enfants jusqu'à ce que ceux-ci soient capables de les exercer (Ministère de l'Éducation, du Loisir et du Sport, 2008). Mais la préservation de l'école comme institution où les élèves peuvent débattre librement et être exposés au pluralisme est indispensable à l'exercice futur d'un choix personnel par les jeunes femmes (McLaughlin, 1992). De plus, les deux parties sont souvent en désaccord sur le moment où les parents devraient cesser d'exercer leurs droits au nom des adolescents.

\section{L'apport d'une perspective multivoque sur l'ethnicité}

Les conceptions univoques de l’ethnicité ont tendance à réifier la réalité comme une donnée ou, si elles adoptent une perspective constructiviste, à réduire son émergence et sa reproduction soit aux rapports de pouvoir entre les groupes dominants et les groupes dominés, soit à l'action d'un sujet autonome. L'analyse menée plus haut illustre clairement la nécessité de dépasser les limites de ces perspectives, tout en reconnaissant que chacune d'entre elles peut fournir, selon la spécificité des contextes, un éclairage essentiel à la compréhension de la dynamique des rapports ethniques.

La première tâche à cet égard est probablement de donner plus de poids dans nos analyses à ce que Juteau (2000) a si bien nommé la frontière interne de l'identité ethnique. En effet, postuler que le minoritaire est défini et redéfini par son rapport au majoritaire ne devrait pas nous amener à penser qu'il a été créé par ce dernier, ni que la différence culturelle ou l'attachement communautaire ne sont que des épiphénomènes de cette relation. À ce titre, malgré les mises en garde énoncées plus haut quant à la nature spécifique du marqueur religieux, ce courant redonne également une légitimité certaine au paradigme interculturel souvent mis à mal par les constructivistes hétérocentristes. Nous avons également besoin, comme le font les interactionnistes sociaux, de réaffirmer le rôle central du sujet et son autonomie dans ce processus dynamique. Cependant, l'individu ne peut être considéré 
comme le producteur de l'ethnicité, mais plutôt comme un médiateur dont l'influence est maximale lors du point de contact entre les frontières internes et externes de l'ethnicité. Les stratégies diverses qu'il choisit de privilégier, que la psychologie sociale et l'anthropologie ont bien explorées (entre autres, les travaux de Camilleri et coll., 1990), ne se développent pas dans un « vacuum social».

Cette troisième perspective qu'on pourrait nommer " l'ethnicité, comme triple produit de la frontière externe, de la frontière interne et de la médiation par le Sujet " est aujourd'hui explorée par de nombreux travaux de nature théorique ou analytique (Bilge, 2009, 2010; Jenkins, 2008; Juteau, 2008; Malesevic, 2004; Rex, 2006) qui illustrent sa pertinence dans la compréhension de la complexité et l'intersectionnalité des processus à l'étude. Sur le plan normatif, cette perspective correspond également étroitement au dilemme de la citoyenneté dans une société pluraliste, qui tente de reconnaître la réalité des inégalités et des exclusions ainsi que la légitimité d'une diversité multiforme, tout en préservant l'indépendance morale et l'autonomie des individus (Bader, 2007; Benhabib, 2002; Eid, Bosset, Milot et Lebel-Grenier, 2009; Weinstock, 2007). Cependant, elle a ses limites. En effet, sa complexité en fait un instrument davantage descriptif que prédictif et, à l'opposé des autres courants, elle se déclare d'emblée incapable de statuer dans une "Grand Theory " sur la priorité relative des trois dynamiques qui jouent dans la production de l'ethnicité, ce qui fait qu'elle doit se limiter à rendre compte de situations contextualisées de rapports ethniques. Sur le plan normatif, elle est également beaucoup moins sécurisante que les perspectives univoques, mais peut-être faut-il s'en réjouir.

En ce qui concerne les politiques et les pratiques scolaires, parce qu'elle reconnaît que l'ethnicité est influencée par des facteurs internes et externes, et que les différences culturelles existent en dehors des relations de pouvoir, cette perspective multivoque justifie à la fois le respect du pluralisme et le développement d'un rapport critique aux inégalités sociohistoriques qui l'ont fait émerger et contribuent encore aujourd'hui à sa définition. Elle peut donc, selon la spécificité des contextes, légitimer aussi bien des interventions visant le maintien des langues et des cultures des minorités et diverses activités multiculturelles ou interculturelles qu'une pédagogie de la conscientisation qui questionne la pertinence même des identités ethnoculturelles traditionnelles.

Par rapport à la question spécifique du port du hijab, adopter une position complexe et contextualisée sur la production de l'ethnicité nous amène d'abord à reconnaître les sens variés de cette pratique. Celle-ci peut relever de toutes les interprétations que nous avons décrites plus haut et même davantage : un geste " naturel » accompli par une femme spontanément et sans questionnement en tant que partie intégrante de sa culture ou de sa religion; une protestation contre l'état actuel de l'intégration des musulmans dans différents pays d'immigration ou leur traitement dans les médias internationaux; un choix profondément réfléchi dans un processus de développement spirituel et social ou enfin une contrainte imposée aux jeunes femmes par une culture patriarcale ou par des penseurs fondamentalistes utilisant cet enjeu dans leur lutte contre le monde occidental. De plus, très souvent ces réalités se juxtaposent chez un même individu. Sur le plan symbolique, il est donc vrai, la plupart du temps, qu'une femme porte plus d'un hijab.

Une perspective multivoque sur l'ethnicité implique aussi que l'accommodement des pratiques religieuses à l'école repose sur la recherche d'un équilibre entre trois objectifs sociaux souvent complémentaires mais parfois opposés: le maintien du pluralisme, la promotion de l'égalité (incluant celle des femmes) et la liberté des individus à définir leurs attachements communautaires. Elle reconnaît donc qu'il est nécessaire que la scolarisation contribue au développement de la culture, de la langue et de la religion des élèves appartenant à des minorités, tout en limitant cette obligation dans une perspective de droits de la personne, perspective qui met en avant l'importance du sujet comme médiateur entre les frontières internes et les frontières externes de l'ethnicité ainsi que la fonction de l'école comme lieu privilégié d'apprentissage de la liberté d'expression et d'exposition au pluralisme.

\section{Conclusion : quelques balises pour l'action}

Lorsqu'on adopte une position multivoque sur la nature de l'ethnicité comme triple produit de la frontière externe, de la frontière interne et de la médiation par le sujet, tout en gardant en tête les limites de chacune des perspectives univoques, il est possible de formuler les balises suivantes pour guider l'action des décideurs et des intervenants en matière d'accommodement de la diversité religieuse à l'école. 
- Une obligation claire des écoles de respecter le droit des parents et des élèves à exprimer leurs croyances religieuses dans l'espace public, quel que soit le sens que l'on attribue à diverses pratiques à cet égard, à moins qu'il n'existe à cette règle des exceptions fondées légalement. À cet égard, le personnel scolaire devrait éviter tout spécialement d'imposer sa propre définition du hijab «acceptable » et éviter d'entretenir des attentes démesurées sur le seuil légitime de la « liberté authentique ».

- $\quad$ La nécessité de définir les exceptions à ce principe dans une perspective de droits de la personne. En ce qui concerne plus spécifiquement l'accommodement religieux, il existe un large consensus sur les balises suivantes, même si leur pertinence face à chaque contexte doit être examinée (Commission des droits de la personne du Québec, 1995; Commission ontarienne des droits de la personne, 1996; Ministère de l'Éducation nationale, 1989; US Department of Education, 1999). Tout d'abord, il faut refuser toute pratique qui aurait un impact discriminatoire sur l'égalité d'accès à l'éducation pour les jeunes filles issues des groupes minoritaires. Deuxièmement, il faut maintenir un climat social exempt de prosélytisme qui garantisse l'exercice d'une certaine liberté de choix par les parents et les élèves face aux pratiques religieuses. Enfin, il faut protéger la sécurité et la santé de l'ensemble des élèves.

- Un engagement équivalent à protéger le droit de l'individu à ne pas se conformer aux attentes de sa communauté en matière de pratiques religieuses. Les écoles, plus que toute autre institution, doivent être protégées de l'abscription communautaire ou du traitement différentiel des élèves à partir d'identités ethniques imposées et essentialisées. Cependant, lorsque le conflit à cet égard oppose les parents à leurs enfants, les écoles devraient mettre l'accent sur leur rôle dans la résolution des conflits de valeurs, et ce, tant entre les parties qu'auprès de l'élève lui-même, souvent déchiré entre deux univers culturels (Hohl et Normand, 1997). Il faut éviter d'entretenir des fantaisies émancipatoires, comme c'est souvent le cas chez les enseignantes (Hohl, 1996), car on tombe alors dans le piège de réduire les appartenances ethniques aux relations de pouvoir et à l'oppression interne au groupe, une position dont les limites ont été clairement illustrées plus haut.

- La nécessité, dans un contexte où le relativisme culturel et cognitif est parfois populaire, de préserver pleinement la fonction spécifique de l'école qui consiste à permettre à tous les élèves d'avoir accès à un savoir critique et d'être exposés à des valeurs différentes de celles de leur communauté d'origine, tout particulièrement dans l'enseignement de l'histoire et de l'éducation à la citoyenneté et dans l'enseignement culturel des religions. Il faut donc que les autorités et le personnel scolaire établissent clairement que l'acceptation par l'institution de l'expression des croyances religieuses par un individu n'implique pas un engagement d'appuyer toutes les valeurs qui peuvent lui être associées, et ce, tout particulièrement lorsque celles-ci peuvent sembler incompatibles avec les principes démocratiques fondamentaux.

- Une prise de conscience accrue chez les décideurs et les éducateurs, mais aussi chez les parents musulmans qui ne sont pas toujours d'accord avec les pratiques religieuses de leur enfant, que ce qui peut sembler un enjeu local ne peut être compris en dehors du contexte plus large de l'islamophobie internationale. Que cela nous plaise ou non, la frontière externe de leur identité ethnique est renforcée chaque jour chez les jeunes adolescents musulmans. Dans un tel contexte, le concept de choix personnel devient encore plus complexe et la distinction entre un hijab religieux et un hijab politique plus confuse. C'est une réalité dont toute discussion en classe sur la question doit tenir compte.

- $\quad$ Finalement, il faut imposer des balises claires durant les débats à l'école, afin que les élèves ne versent pas dans des dérives démagogiques ou racisantes, explicites ou déguisées en "préoccupations démocratiques ». Les discours essentialistes qui opposent le "Nous » et le "Eux » présentés comme des réalités homogènes et dichotomiques doivent être tout particulièrement évités. La présentation stéréotypée de la majorité des musulmans comme orthodoxes ou même terroristes, ou des femmes musulmanes comme des victimes passives de la manipulation des hommes, n'est certainement pas un atout dans le développement d'une société cohésive et égalitaire, quelle que soit la position qu'on adopte sur la légitimité de l'accommodement religieux à l'école publique. 


\section{Références bibliographiques}

Abdallah-Pretceille, M. (1992). Quelle école pour quelle intégration? Paris : Hachette.

Amara, F. (2003). Ni Putes, Ni Soumises. Paris : La Découverte.

Amiraux, V. (2010a). De l'Empire à la République : I'Islam de France. Dans N. Bancel, F. Bernault, P. Blanchard, A. Boubeker, A. Mbembe et F. Vergès (dir.), Rupture postcoloniale. Les nouveaux visages de la société française (p. 379-390). Paris : La Découverte.

Amiraux, V. (2010b). Suspicion publique et gouvernance de l'intime : contrôle et surveillance des populations musulmanes dans I'Union européenne. Dans D. Bigo, E. Guillet et A. Scherrer (dir.), Mobilités sous surveillance. Perspectives croisées Union européenne-Canada (p. 73-87). Montréal : Athéna.

Bader, V. (2007). Secularism or democracy? Associational governance of religious diversity. Amsterdam : Amsterdam University Press.

Barth, F. (1969). Ethnic groups and boundaries. Boston : Little, Brown and Co.

Baubérot, J. (2007). Les laïcités dans le monde. Paris : Presses Universitaires de France.

Beauchemin, J. (2003). Qu'est-ce qu'être Québécois : entre la préservation de soi et l'ouverture à l'autre. Dans A. Gagnon (dir.), Québec, État et société (p. 27-45). Montréal : Québec Amérique.

Beiner, R. (1995). Theorizing citizenship. New York : State University of New York Press.

Benhabib, D. (2011). Ma vie à contre-Coran. Montréal : VLB Éditeur.

Benhabib, S. (2002). The claims of culture : Equality and diversity in the global era. Princeton : Princeton University Press.

Berger, P. et Luckman, T. (1967). The social construction of reality. Hardmondsworth : Allen Lane, Pergamon Press.

Bernard, P. (1995, 12 juillet). Le Conseil d'État refuse l'interdiction totale du foulard islamique à l'école. Le Monde.

Bilge, S. (2008). Between gender and cultural equality. Dans E. Isin (dir.), Recasting the Social in Citizenship (p. 100133). Toronto : University of Toronto Press.

Bilge, S. (2009). Théorisation féministe de l'intersectionnalité : débats et approches. Diogène, revue internationale des sciences humaines, 225, 158-176.

Bilge, S. (2010). De l'analogie à l’articulation : théoriser la différenciation sociale et l'inégalité complexe. L'homme et la société, 176-177, 43-64.

Bosset, P. (2010). «Concevoir juridiquement la liberté dans une société multiculturelle ». Dans P. Leprecht et J. Ayoub (dir.), Le sens de la liberté. Québec : Presses de l’Université Laval.

Bouchard, G. et Taylor, C. (2008). Fonder l'avenir. Le temps de la conciliation. Rapport du Comité de consultation sur les pratiques d'accommodement reliées aux différences culturelles. Québec : Gouvernement du Québec

Bourgeault, G., Gagnon, F., Mc Andrew, M. et Pagé, M. (1995). L'espace de la diversité culturelle et religieuse à l'école dans une démocratie de tradition libérale. Revue européenne des migrations internationales, 11(3), 79-103.

Bourgeault, G. et Pietrantonio, L. (1996). L'école dans une société pluraliste et « l'indépendance morale des individus ». Dans F. Gagnon, M. Mc Andrew et M. Pagé (dir.), Pluralisme, citoyenneté et éducation (p. 231254). Montréal/Paris : L'Harmattan.

Bourhis, R. et Harvey, S. (2010). In-group allocation bias. Dans J. Levine et M. Hogg (dir.), Encyclopedia of Group Process and Intergroup Relations. Thousand Oaks, CA : Sage.

Bourhis, R. et Leyens, J.-J. (dir.) (1994). Stéréotypes, discrimination et relations intergroupes. Liège : Mardaga. 
Boyd, M. (2007). Religion-based alternative diffuse resolution : A challenge to multiculturalism. Dans K. Banting, T. Courchesne et S. Seidle (dir.), Belonging? Diversity, recognition and shared citizenship in Canada. Montréal : The Art of the State/RPP.

Bramadat, P. et Seljak, O. (2009). Religion and ethnicity in Canada. Toronto : Toronto University Press.

Brodeur, P. (2010). Religious pluralism in the light of American Muslim identities. Dans Z. Hirji (dir.), Diversity and pluralism in Islam : Historical and contemporary discourses among Muslims (p. 63-84). London : I. D. Torist.

Buyck, M. et Fall, K. (1995). L'intégration des immigrants au Québec : Des variations de définition dans un échange oral. Sillery : Les Éditions du Septentrion.

Burawoy, M., Blum, J., George, S., Gille, Z., Thayer, M., Gowan, T., Haney, L., Klawiter, M., Lopez, S. et Riain, S. (2000). Global ethnography: forces, connections and imaginations in post-modern world. Berkeley : University of California Press.

Camilleri, C., Kasterszteyn, J., Lipiansky, E., Malewska-Peyre, H., Taboada-Leonetti, I. et Vasquez, A. (1990). Stratégies identitaires. Paris : Presses Universitaires de France.

Cicéri, C. (1999). Le foulard islamique à l'école publique: analyse comparée du débat dans la presse française et québécoise francophone (1994-1995). Mémoire de maîtrise présentée à la Faculté des sciences de l'éducation, Université de Montréal.

Commission des droits de la personne du Québec (1995). Le pluralisme religieux au Québec : un défi d'éthique sociale. Document soumis à la réflexion publique. Montréal : Gouvernement du Québec.

Commission ontarienne des droits de la personne (1996). Politique sur la croyance et les mesures d'adaptation relative aux observances religieuses. Toronto.

Dei, G. (1996). Antiracist education : Theory and practice. Halifax : Fernwood Publishing.

Deschamps, J.-C. et Devos, T. (1999). Les relations entre identités individuelles et collectives ou comment la similitude et la différence peuvent covarier. Dans J.C. Deschamps, J.F. Morales, D. Paers et F. Worchel (dir.), L'identité sociale : la construction d'individus dans les relations entre groupes (p. 149-167). Grenoble : Presses universitaires de Grenoble.

Dortier, J.-F. et Testo, L. (dir.) (2005). Dieu ressuscité. Les religions face à la modernité. Sciences humaines, 160.

Eid, P., Bosset, P., Milot, M. et Lebel-Grenier, S. (2009). Appartenance religieuse, appartenance citoyenne : un équilibre en tension. Québec : Presses de l'Université Laval.

Fekete, L. (2006). Enlightened fundamentalism? Immigration, feminism and rights. Race and Class, 48(2), 1-22.

Fontaine, L. (1993). Un labyrinthe carré comme un cercle. Enquête sur le ministère des Communautés culturelles et de l'Immigration et sur ses acteurs réels et imaginaires. Montréal : Éditions l'Étincelle.

Forum musulman canadien (1999). Mémoire présenté à la Commission parlementaire de l'éducation, Assemblée nationale du Québec. Montréal : Forum musulman canadien.

Freitag, M. (1981). Théorie marxiste et réalité nationale. Pluriel, 26, 3-38.

Gallagher, T. (2004). Education in divided societies. Basingstoke : Palgrave Macmillan.

Gaudet, J. (1994, 28 septembre). Des principes fondamentaux sont en jeu dans le débat sur le foulard islamique. La Presse

Gautherin, J. (2000). L'universalisme laïque à l'épreuve. Dans A. Van Zanten (dir.), L'école, l'état des savoirs. Paris : La Découverte.

Geadah, Y. (1996). Femmes voilées, intégrismes démasqués. Montréal : VLB Éditeur. 
Germain, A., Liégeois, L. et Hoernig, H. (2007). L'espace public à l'épreuve des religions : des paysages pluriels à négocier ? Dans A. Da Cunha et L. Matthey (dir.), La ville et I'urbain : des savoirs émergents (p. 321-340). Lausanne : Presses polytechniques et universitaires romandes.

Gil-White, F. (2001). Are ethnic groups biological "species" to the human brain? Current Anthropology, 42(4), 515554.

Gimenez, M. (2001). Marxism and class, gender and race : Rethinking the trilogy. Race, Gender and Class, 8(2), 2333.

Giroux, H. (1981). Hegemony, resistance and the paradox of educational reform. Dans H. Giroux, A. Penna et W. Pinar (dir.), Curriculum and instruction : Alternatives in education (p. 400-425). Berkeley, CA : McCutchan Publishing.

Glenn, J. et De Jong, E. (1996). Educating immigrant children : Schools and language minorities in twelve nations. New York : Garland.

Guillaumin, C. (1977). Race et nature : systèmes de marques, idées de groupe naturel et rapports sociaux. Pluriel, 11, 39-55.

Guttman, A. (1987). Democratic education. Princeton, NJ : Princeton University Press.

Hall, S. (1980). Cultural studies : Two paradigms. Media, Culture and Society, 2, 57-72.

Halstead, J. (1986). The case for Muslim voluntary aided schools : Some philosophical reflexions. London : The Islamic Academy.

Hargreaves, D. (1996). Diversity and choice in school education : A modified libertarian approach. Oxford Review of Education, 22(2).

Helly, D. (1992). L'immigration pour quoi faire ? Québec : Presses de l’Université Laval.

Hohl, J. (1996). Résistance à la diversité culturelle au sein des institutions scolaires. Dans M. Pagé, M. Mc Andrew et F. Gagnon (dir.), Pluralisme, citoyenneté et éducation (p. 337-247). Montréal/Paris : L'Harmattan.

Hohl, J. et Cohen-Émerique, M. (1999). La menace identitaire chez les professionnels en situation interculturelle : le déséquilibre entre scénario attendu et scénario reçu. Études ethniques au Canada/Canadian Ethnic Studies, 31(1), 106-123.

Hohl, J. et Normand, M. (1997). Construction et stratégies identitaires des enfants et des adolescents en contexte migratoire : le rôle des intervenants scolaires. Revue française de pédagogie. L'école et la question de I'immigration, 117, 39-52.

Holmes, B. (1981). Comparative education : A study of educational factors and traditions. London : Routledge and Kegan Paul.

Hoerder, D. (2000). Creating societies : Immigrant lives in Canada. Montréal : McGill-Queen's University Press.

Jenkins, R. (2008). Rethinking ethnicity : Arguments and explorations ( $2^{\mathrm{e}}$ edition). Thousand Oaks, CA : Sage Publications

Juteau, D. (1993). The production of the Québécois nation. Humboldt Journal of Social Relations, 19(2), 79-108.

Juteau, D. (2000). L'ethnicité et ses frontières. Montréal : Presses de l'Université de Montréal.

Juteau, D. (2006). Forbidding ethnicities in French sociological thought : The difficult circulation of knowledge and ideas. Mobilities, 1(3), 391-408.

Juteau, D. (2008). Multicultural citizenship beyond recognition. Dans E. Isin (dir.), Recasting the social in citizenship (p. 69-98). Toronto : University of Toronto Press.

Kanouté, F., Lavoie, A. et Duong, L. (2004). L'interculturel et la formation des enseignants. Éducation Canada, 44(2), 8-10 et 54.

Alterstice - Revue Internationale de la Recherche Interculturelle, vol. 1, $n^{\circ} 1$ 
Kepel, G. (1992). L'intégration suppose que soit brisée la logique communautaire. Dans C. Wihtol de Wenden et A.-M. Chartier (dir.), École et intégration des immigrés, Paris, La documentation française [Dossiers d'actualité mondiale $n^{\circ}$ 693].

Kurd, R. (1995, 15 février). My hijab is an act of worship - and none of your business. The Globe and Mail. Kymlicka, W. (1995). Multicultural citizenship. Oxford : Clarendon Press.

Kymlicka, W. (2000). Les droits des minorités et le multiculturalisme : I'évolution du débat anglo-américain. Comprendre, 1, 141-171.

Kymlicka, W. et Norman, W. (dir.) (2000). Citizenship in diverse societies. Oxford : Oxford University Press.

Laperrière, A., avec la collaboration de Majid D'Khissy, René Dolce, Nicole Fleurant Et Lejacques Compere. (1991). "De l'indifférenciation à l'évitement ». Dans F. Ouellet et M. Pagé (dir.), Pluriethnicité, éducation et société : construire un espace commun (p. 542-562). Montréal : Institut québécois de recherche sur la culture.

Leblanc, M., Fortin, S. et Le Gall, J. (2008). « Entre la oumma, l'ethnicité et la culture : le rapport à l'Islam chez les Musulmans francophones de Montréal ». Diversité urbaine, 8(2), 99-134.

Lorcerie, F. (1996). À propos de la crise de la laïcité en France : dissonance normative. Dans F. Gagnon, M. Mc Andrew et M. Pagé (dir.), Pluralisme, citoyenneté et éducation (p. 121-136). Montréal/Paris : L'Harmattan.

Lorcerie, F. (2003). L'école et le défi ethnique. Éducation et intégration. Paris : INRP-ESF, collection Actions Sociales/Confrontations.

Lorcerie, F. (2005). A l'assaut de l'agenda public. La politisation du voile islamique en 2003-2004. Dans F. Lorcerie (dir.), La politisation du voile. L'affaire en France, en Europe et dans le monde arabe (pp. 11-36). Paris : L'Harmattan.

Malesevic, S. (2004). Sociological theory and ethnic relations: Where to go from here? Dans S. Malesevic, The sociology of ethnicity (p. 160-194). Londres : Sage.

Mc Andrew, M. (1996). Diversité culturelle et religieuse : divergences des rhétoriques, convergences des pratiques? Dans F. Gagnon, M. Mc Andrew et M. Pagé (dir.), Pluralisme, citoyenneté et éducation (p. 287320). Montréal/Paris : L'Harmattan.

Mc Andrew, M. (2001). Immigration et diversité à l'école. Le débat québécois dans une perspective comparative. Montréal : Presses de l’Université de Montréal.

Mc Andrew, M. (2003a). School spaces and the construction of ethnic relations : Conceptual and policy debates. Canadian Ethnic Studies/Études ethniques au Canada, 35(2), 14-29.

Mc Andrew, M. (2003b). L'accommodement raisonnable : atout ou obstacle dans l'accomplissement des mandats de l'école? Options CSQ, 22 (automne), 131-147.

Mc Andrew, M. (2010). Les majorités fragiles et l'éducation : Belgique, Catalogne, Irlande du Nord, Québec. Montréal : Presses de l'Université de Montréal.

Mc Andrew, M., Cicéri, C. et Jacquet, M. (1997). La prise en compte de la diversité culturelle et religieuse dans les normes et pratiques de gestion des établissements scolaires : une étude exploratoire dans cinq provinces canadiennes. Revue des sciences de l'éducation, 23(1), 209-232.

Mc Andrew, M., Milot, M., Imbeault, J. et Eid, P. (dir.) (2008). L'accommodement raisonnable et la diversité religieuse à l'école publique : normes et pratiques. Montréal : Éditions Fides.

McLaughin, T. (1992). Citizenship, diversity, and education, a philosophical perspective. Journal of Moral Education, 21(3), 235-250.

Milot, M. (2009b). The psychology of extreme beliefs. Dans M. St-Yves et M. Tanguay (dir.), The psychology of criminal investigations. The search of the truth (p. 21-34). Vancouver/Toronto : UBC Press. 
Milot, M. (2010). Conceptions of the good : Challenging premises of delibrative democracy in practice. Dans D. Kahane, D. Weinstock, D. Leudet et M. Williams (dir.), Deliberative democracy in practice (p. 21-34). Vancouver/Toronto : UBC Press.

Milot, M. et Koussens, D. (dir.). (2009). Reconnaissance de la diversité religieuse : débats actuels dans différentes sociétés. Diversité urbaine, 9(1).

Milot, M., Portier, P. et Willaime, J.-P. (2010). Pluralisme religieux et citoyenneté. Rennes : Presses universitaires de Rennes.

Ministère de l'Éducation, du Loisir et du Sport (2008). Module de formation à l'intention des gestionnaires. La prise en compte de la diversité culturelle et religieuse en milieu scolaire : de la théorie à la pratique. Québec : MELS.

Ministère de l'Éducation nationale (1989). Avis du Conseil d'État et déclaration du Ministre concernant le port de signes d'appartenance à une communauté religieuse dans les établissements scolaires. Paris : MEN.

Ministère de l'Éducation nationale, de l'Enseignement supérieur et de la Recherche (2004). Loi $n^{\circ} 2004-228$ du 15 mars 2004 encadrant, en application du principe de laïcité, le port de signes ou de tenues manifestant une appartenance religieuse dans les écoles, collèges et lycées publics. Circulaire du 18 mai 2004 relative à la mise en œuvre. Paris : MENESR.

Payet, J.-P. et Giuliani, F. (2010). Du tabou au débat : le problème de la ségrégation ethnique dans l'école française. Dans M. Mc Andrew, M. Milot et A. Triki-Yamani (dir.), L'école et la diversité. Perspectives comparées (p. 171-180). Québec : Presses de l’Université Laval.

Potvin, M. (2008). Crise des accommodements raisonnables. Une fiction médiatique ? Montréal : Athéna Éditions.

Probyn, E. (1987). Bodies and antibodies : Feminism and the post-modern. Cultural Studies, 1(3), 340-360.

Rex, J. (2006). Les fondamentaux d'une théorie de l'ethnicité. Ethnicité et citoyenneté, la sociologie des sociétés multiculturelles. Paris : L’Harmattan.

Ramadan, T. (1994). Les musulmans dans la laïcité. Responsabilités et droits des musulmans dans les sociétés occidentales. Lyon : Éditions Tawhid.

Sarup, M. (1988). An introductory guide to post-structuralism and postmodernism. New York : Harvester Wheatsheaf.

Sarwar, G. (1994). British Muslims and schools. London : Muslim Educational Trust.

Shadid, W. et Van Koningsveld, P. (dir.) (1996). Muslims in the margin. Political responses to the presence of Islam in Western Europe. Kampen : Kok Pharos.

Schnapper, D. (1991). La France de l'intégration. Paris : Gallimard.

Stasi, B. (2003). Rapport au président de la République. Commission de réflexion sur l'application du principe de laïcité dans la République. Paris : La documentation française.

Taboada-Leonetti, I. (1990). Stratégies identitaires et minorités : le point de vue du sociologue. Dans C. Camilleri, J. Kasterszteyn, E. Lipiansky, H. Malewska-Peyre, I. Taboada-Leonetti et A. Vasquez (dir.), Stratégies identitaires (p. 43-83). Paris : Presses Universitaires de France.

Taguieff, P. (1987). La force du préjugé. Essai sur le racisme et ses doubles. Paris : La Découverte.

Tajfel, H. (1982). Human groups and social categories. Cambridge : Cambridge University Press.

Touraine, A. (1997). Pour vivre ensemble, égaux et différents. Paris : Fayard.

Willey, R. (1999). US Department of Education Secretary's statement on religious expression. Récupéré du site du gouvernement : www2.ed. gov/Speeches/08-1995/religion.html

Van Den Berghe, P. (1967). Race and racism : A comparative perspective. New York : John Wiley and Sons.

Alterstice - Revue Internationale de la Recherche Interculturelle, vol. 1, $n^{\circ} 1$ 
Venel, N. (2004). Musulmans et citoyens. Paris : Presses Universitaires de France.

Verlot, M. (2002). Resistance, complexity and the need for rethinking intercultural education. Kolor. Journal of Moving Communities, 1, 5-19.

Vermeulen, H. et Govers, C. (1996). Introduction. In H. Vermeulen et C. Govers (dir.), The anthropology of ethnicity, beyond ethnic groups and boundaries (p. 1-11). Amsterdam : Het Spinhuis Publishers.

Weinstock, D. (2007). Strategic spatial essentialism : Latin-Americans real and imagined geographies belonging in Toronto. Social and Cultural Geography, 8(3), 455-473.

Woehrling, J. (2008). Les fondements et les limites de l'accommodement raisonnable en milieu scolaire. Dans M. Mc Andrew, M. Milot, S. Imbeault et P. Eid (dir.), L'accommodement raisonnable et la diversité religieuse à l'école publique. Normes et pratiques (p. 43-53). Montréal : Fides.

Zylberberg, J. (1994). Le nationalisme québécois. De l'ethnoreligion à la religion étatique. La pensée et les hommes, 27, 93-98. 